\title{
Gastric Metastasis Mimicking Linitis Plastica 20 Years after Primary Breast Cancer. A Case Report
}

\author{
Noelle Asmar ${ }^{1}$, Jean-Francois Rey ${ }^{1}$, Christophe Sattonnet ${ }^{2}$, Jerome Barriere ${ }^{3}$
}

1) Department of

Gastroenterology and

Digestive Endoscopy, Arnault Tzanck Institute Saint-Laurent du Var,

2) DIAG Pathologie, Nice,

3) Department of Medical

Oncology, Pôle de Santé Saint Jean, Cagnes-sur-Mer, France

Address for correspondence: Noelle Asmar

Department of

Gastroenterology and

Digestive Endoscopy, Arnault Tzanck Institute 231 Avenue Docteur Maurice Donat 06700 Saint-Laurent du Var, France noelle.asmar@hotmail.com

Received: 17.10.2018 Accepted: 17.11.2018

\section{ABSTRACT}

Breast cancer metastases to the gastrointestinal tract are rare, with a median time interval from the diagnosis of the primary tumor to metastasis up to 7 years. The stomach is the most frequent metastatic site and invasive lobular carcinoma is the type with the highest affinity to the digestive system. We report the case of an 84-yearold female patient, with a past medical history 20 years earlier of invasive lobular carcinoma of the breast, who presented for dyspepsia. Upper endoscopy revealed hypertrophic gastric folds compatible with primary linitis plastica. Histology showed proliferation of malignant poorly cohesive cells. Immunohistochemistry stain showed intense positivity of estrogen receptors and anti-GATA-binding protein 3 nuclear antibodies, and absence of the human epidermal growth factor receptor 2. These findings confirmed the diagnosis of a metachronous metastasis of the invasive lobular breast adenocarcinoma. Considering metastases from breast cancer is crucial when patients with any subtle gastric symptom and a past medical history of invasive lobular adenocarcinoma of the breast seek medical advice, even though more than 20 years after primary breast cancer. Immunohistochemistry is the key to final diagnosis as these lesions can endoscopically and histologically mimic primary linitis plastica.

Key words: breast carcinoma - metastases - linitis plastica - estrogen receptors - anti-GATA-binding protein 3 (GATA3) nuclear antibodies.

Abbreviations: CK: cytokeratin; ER: estrogen receptors; EUS-FNA: endoscopic ultrasound-fine needle aspiration; GATA3: GATA-binding protein 3; GCDFP-15: gross cystic disease fluid protein-15; HER2: human epidermal growth factor receptor 2; IHC: immunohistochemistry; PR: progesterone receptors.

\section{INTRODUCTION}

Breast cancer is the most common type of cancer in women and despite combination of therapies it might relapse in more than half of the cases [1]. The time interval between initial diagnosis and distant metastases can be up to many years [2].

Metastases to the gastrointestinal tract are rare and do not exceed $5 \%$ of the cases [3] with the stomach being the most frequent metastatic site $[4$, 5]. Although gastric metastases from primary gynecological tumors are scarce, those from breast cancers seem to be more frequent than those from the uterus or the ovaries [6]. Lobular carcinoma of the breast has more affinity to the gastrointestinal tract than the other types [4], with an incidence of $4.5 \%$ compared to $0.2 \%$ in ductal carcinoma [7]. The mean time interval between the diagnosis of primary breast cancer and the appearance of gastric metastases usually ranges from 5 to 7 years $[2,6,8]$.

\section{CASE REPORT}

Herein, we report the case of an 84-year-old female patient who presented to our department for dyspeptic symptoms. In her past medical and surgical history we noted a left mastectomy 20 years ago for lobular carcinoma of the breast that was estrogen receptors (ER) positive, progesterone receptors (PR) and human epidermal growth factor receptor 2 (HER2) negative. She received adjuvant chemotherapy, radiotherapy and hormonal therapy. She was in complete remission on follow up and had surveillance with a yearly mammography. Her last mammography performed two months prior to her current presentation did not show any abnormalities. Her 
symptoms started few months before seeking medical advice. She complained of dyspepsia and gastric fullness. She had no nausea, vomiting or epigastric pain.

On physical examination she had no palpable masses in the epigastrum or elsewhere in the abdomen, had no palpable nodules in her right breast and no palpable lymph nodes.

Gastroscopy revealed thickened gastric folds and diffuse infiltration of the stomach wall involving the fundus. There were no nodules, erosions, ulcers or stigmata of hemorrhage (Fig. 1). Multiple mucosal blinded biopsies were taken.

Pathology showed proliferation of malignant, nonmucinous, poorly cohesive cells in a fibro- inflammatory stroma suggesting the diagnosis of primary, signet ring cell type, gastric adenocarcinoma. Given the past medical history, immunohistochemical analysis was done. Estrogen receptors were highly positive while PR and HER2 were negative. Cytokeratines (CK) 5 and 20 were negative and CK7 was positive. Anti- GATA-binding protein 3 (GATA3) nuclear antibodies were significantly positive on immunohistochemistry (IHC) staining and together with the previous findings confirmed the diagnosis of a metachronous metastasis of invasive lobular breast carcinoma to the stomach (Fig. 2).

A computer tomography (CT) scan of the thorax and abdomen and a positron emission tomography (PET) scan did not show any other distant sites of metastases.

Hormonotherapy with Fulvestrant 500 mg every 28 days was started and digestive symptoms rapidly resolved.

\section{DISCUSSION}

In a review of 159 gastric metastases from breast adenocarcinoma, the mean age at diagnosis of the metastasis was 47 years whereas the median interval between the primary tumor and the secondary gastric location was 78 months (range, 33-96 months) [6]. Our patient was much older and had her primary breast lobular adenocarcinoma diagnosed 20 years earlier, which makes it an unusual case.

Symptoms are nonspecific and may range from dyspepsia (31\%), loss of appetite, abdominal pain to vomiting and sometimes upper gastrointestinal tract obstruction $[3,6,8]$. Endoscopic findings are also nonspecific, as they may appear as mucosal ulcers, nodules, external compression and diffuse infiltration mimicking linitis plastica [6]. This latter is the most frequently described [9], in up to $73 \%$ of the cases [10].

In our case, the patient had dyspepsia and diffuse infiltrative appearance of the fundus on upper gastrointestinal endoscopy, which could have been thought to be primitive linitis plastica.

In this context, a past medical history of lobular breast cancer led to further workup and, if the initial biopsies would not have been contributory, more biopsies should have been done to get tissue from the deeper layers of the stomach and possibly endoscopic ultrasound-fine needle aspiration (EUSFNA) for a good tissue diagnostic yield. In fact, metastases to the stomach usually spread to the submucosal layer with a diffuse infiltrative pattern on endoscopy, without destruction
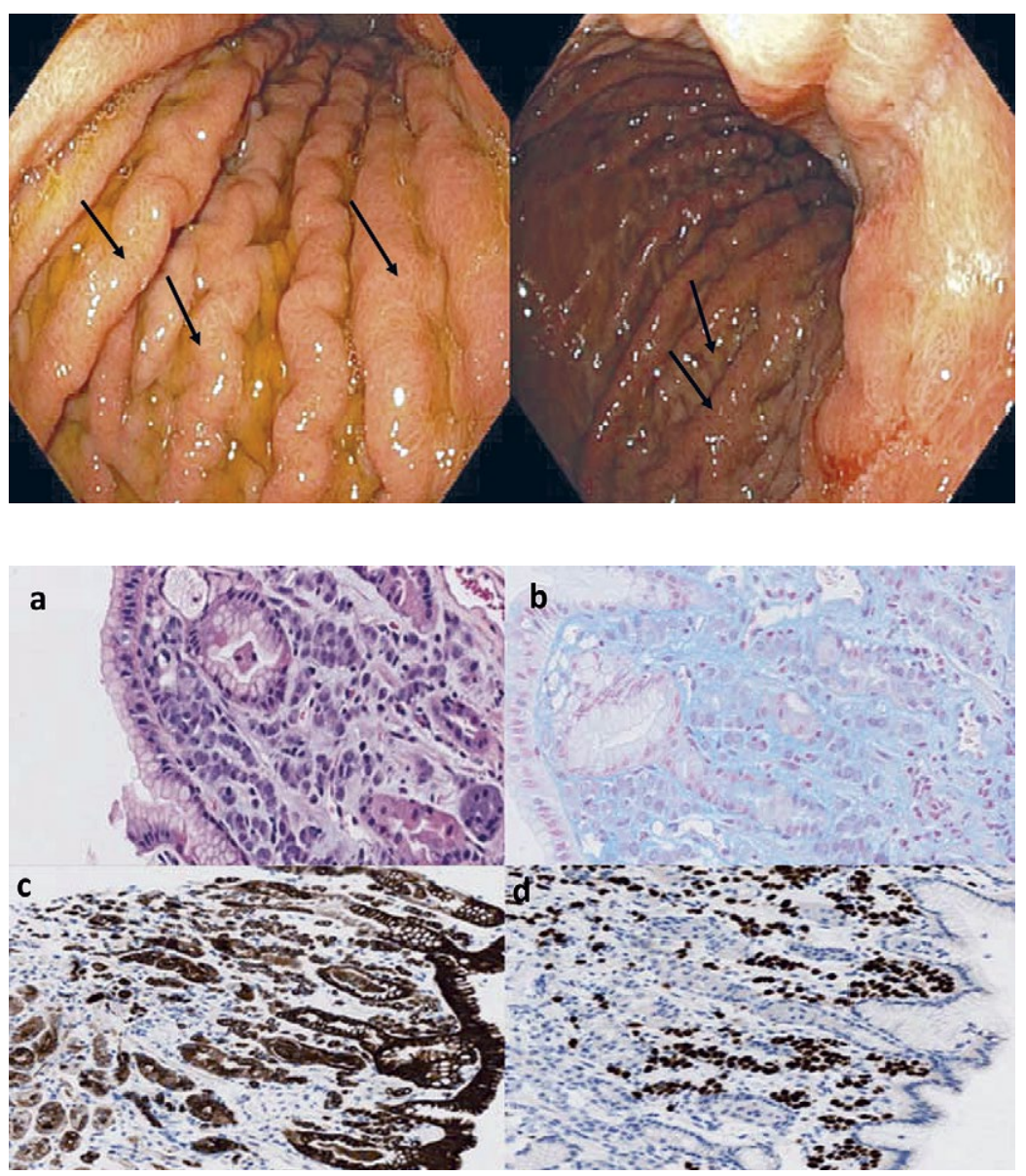

Fig. 1. Upper endoscopy. Hypertrophic gastric folds in the fundus with infiltrative appearance mimicking primary linitis plastica.

Fig. 2. Histological examination of biopsies from the fundus. a) Dense anarchic spread of small basophile cells with increased nuclear to cytoplasmic ratio suggestive of malignant proliferation of discohesive tumor cells (H\&E x40); b) Blue staining mucous cells and clear staining malignant cells prove that the latter do not contain mucus and are not of gastric origin (Alcian Blue $\mathrm{x} 40$ ); c) Immunohistochemistry with cytoplasmic marker Pan cytokeratine shows high positivity consistent with a cellular proliferation of carcinomatous origin; d) Immunohistochemistry with antibodies to nuclear marker GATA3 shows intense stain compatible with breast cells. 
of the mucosa which can give rise to false negative biopsies in almost $10 \%$ of the cases $[6,11]$.

Immunohistochemistry stain is crucial to differentiate primitive gastric cancer from metastatic disease in such cases. Many markers have been used, with ER and PR being mostly examined. Their positivity is suggestive of breast cancer; nevertheless, they can be positive in patients with cancer of gastric origin in $32 \%$ and $12 \%$ of the cases, respectively [12]. Knowing that their intense positivity is consistent with a diagnosis of metastasis of breast cancer [13], this could make the latter the most probable in our case. Human epidermal growth factor receptor 2, which is expressed in $20 \%$ of gastric adenocarcinomas, is actually absent in lobular breast cancers $[2,3]$ and could thus reinforce our suspected diagnosis.

Gross Cystic Disease Fluid Protein-15 (GCDFP-15) and mammaglobin are two other markers that may also be used to characterize metastases from breast cancer, as they are expressed in $32-47 \%$ and $42-87 \%$ of the cases, respectively [14-16]. They have a high specificity, but low sensitivity [17].

Recently, GATA3 emerged as a marker of urothelial and breast cancers. It has $100 \%$ positivity in breast lobular carcinoma and $96 \%$ positivity in breast ductal carcinoma. Only $5 \%$ of gastric malignant tumors stain positive for GATA3 [18].

Our patient had diffuse strong nuclear expression of GATA3 which, along with a past medical history of invasive lobular breast carcinoma, CK5-/CK20-/CK7+, HER2 negativity and ER high positivity, can reliably confirm the diagnosis of a metachronous metastasis to the stomach, from invasive lobular breast adenocarcinoma.

\section{CONCLUSION}

Our case report supports the fact that considering gastric metastases from breast cancer and performing an upper gastrointestinal endoscopy are crucial in a patient presenting with any subtle gastric symptom and having a past medical history of breast adenocarcinoma even more than 20 years ago. If initial endoscopy and biopsies are negative, repeating them assisted by EUS-FNA for deep sampling might be necessary, to insure sufficient tissue yield. Adequate information should then be given to the pathologist for careful review of the specimen and specific IHC studies.

Conflicts of interest: The authors declare no conflict of interest for this article.

Authors' contribution: N.A. designed and wrote the manuscript. J.F.R. performed the upper endoscopy and provided the endoscopic photos. C.S. did the histological diagnosis and provided the pathology photos. J.B. supplied the past and actual oncological history of the patient.

\section{REFERENCES}

1. Signorelli C, Pomponi-Formiconi D, Nelli F, Pollera CF. Single colon metastasisfrom breast cancer. Tumori 2005;91:424-427. doi:10.1177/030089160509100509
2. Villa Guzmán JC, Espinosa J, Cervera R, Delgado M, Patón R, Cordero Garcia JM. Gastric and colon metastasis from breast cancer: case report, review of the literature, andpossible underlying mechanisms. Breast Cancer (Dove Med Press) 2016;9:1-7. doi:10.2147/BCTT.S79506

3. Horimoto Y, Hirashima T, Arakawa A, Miura H, Saito M. Metastatic colonic and gastric polyps from breast cancer resembling hyperplastic polyps. Surg Case Rep 2018;4:23. doi:10.1186/s40792-018-0433-4

4. Jmour O, Belaïd A, Mghirbi F, Béhi K, Doghri R, Benna F. Gastric metastasis of bilateral breast cancer. J Gastrointest Oncol 2017;8:E16-E20. doi:10.21037/jgo.2016.10.03

5. Buka D, Dvořák J, Richter I, Hadzi ND, Cyrany J. Gastric and colorectal metastases of lobular breast carcinoma: a case report. Acta Medica 2016;59:18-21. doi:10.14712/18059694.2016.50

6. Zullo A, Balsamo G, Lorenzetti R, et al. Gastric metastases from gynaecologic tumors: case reports and review of the literature. Ann Transl Med 2016;4:483. doi:10.21037/atm.2016.12.51

7. Woo J, Lee JH, Lee KE, Sung SH, Lim W. Gastric Metastasis as the First Presentation One Year Before Diagnosis of Primary Breast Cancer. Am J Case Rep 2018;19:354-359. doi:10.12659/AJCR.908039

8. Kim DH, Son SM, Choi YJ. Gastric metastasis from invasive lobular breast cancer, mimicking primary gastric cancer: A case report. Medicine (Baltimore) 2018;97:e0258. doi:10.1097/MD.0000000000010258

9. Taal BG, Boot H, van Heerde P, de Jong D, Hart AA, Burgers JM. Primary non- Hodgkin lymphoma of the stomach: endoscopic pattern and prognosis in low versus high grade malignancy in relation to the MALT concept. Gut 1996;39:556-561. doi:10.1136/gut.39.4.556

10. Madeya S, Börsch G. Gastrointestinal metastases of breast carcinoma. Gastrointest Endosc 1993;39:103-104. doi:10.1016/S00165107(93)70029-2

11. Yagi Y, Sasaki S, Yoshikawa A, et al. Metastatic gastric carcinoma from breast cancer mimicking primary linitis plastica: A case report. Oncol Lett 2015;10:3483-3487. doi:10.3892/ol.2015.3788

12. Pectasides D, Psyrri A, Pliarchopoulou K, et al. Gastric metastases originating from breast cancer: report of 8 cases and review ofthe literature. Anticancer Res 2009;29:4759-4763.

13. Yamamoto D, Yoshida H, Sumida K, et al. Gastric tumor from metastasis of breast cancer. Anticancer Res 2010;30:3705-3708.

14. Luo MH, Huang YH, Ni YB, et al. Expression of mammaglobin and gross cystic disease fluid protein-15 in breast carcinomas. Hum Pathol 2013;44:1241-1250. doi:10.1016/j.humpath.2012.10.009

15. Yan Z, Gidley J, Horton D, Roberson J, Eltoum IE, Chhieng DC. Diagnostic utility of mammaglobin and GCDFP-15 in the identification of metastatic breast carcinoma in fluid specimens. Diagn Cytopathol.2009;37:475-478. doi:10.1002/dc.21039

16. Bhargava R, Beriwal S, Dabbs DJ. Mammaglobin vs GCDFP-15: an immunohistologic validation survey for sensitivity and specificity. Am J Clin Pathol 2007;127:103-113. doi:10.1309/ TDP92PQLDE2HLEET

17. Ciulla A, Castronovo G, Tomasello G, et al. Gastric metastases originating from occult breast lobular carcinoma: diagnostic and therapeutic problems. World J Surg Oncol 2008;6:78. doi:10.1186/14777819-6-78

18. Miettinen M, McCue PA, Sarlomo-Rikala M, et al. GATA3: a multispecific but potentially useful marker in surgical pathology: a systematic analysis of 2500 epithelial and nonepithelial tumors. Am J Surg Pathol 2014;38:13-22. doi:10.1097/PAS.0b013e3182a0218f 